\title{
Experimental Study on Transport of Carboxylate Polystyrene Microspheres Using as Cryptosporidium Oocysts Surrogate with Runoff from the Slope Soil to the Surface Water Bodies
}

\author{
Tao Yuan*(***), Sen Cheng**, Lai Zhou**, Qiyan Feng** and Ping Lu**† \\ *Department of Architectural Intelligence, Jiangsu Vocational Institute of Architectural Technology, Xuzhou, Jiangsu \\ 221000, China \\ **Department of Environmental Science and Spatial Informatics, China University of Mining and Technology, \\ Xuzhou 221116, China \\ ***Jiangsu Collaborative Innovation Centre for Building Energy Saving and Construct Technology, Xuzhou 221116, \\ China \\ †Corresponding author: Ping Lu; lupingcumt@126.com
}

\author{
Nat. Env. \& Poll. Tech. \\ Website: www.neptjournal.com \\ Received: 05-06-2019 \\ Accepted: 24-07-2019 \\ Key Words: \\ Cryptosporidium surrogate; \\ Runoff factors; \\ Transport of carboxylate \\ polystyrene; Slope soil
}

\begin{abstract}
Cryptosporidium can transport from the soil to the water resulting in the contamination of the surrounding water bodies. However, there are few pieces of research on the transport of Cryptosporidium from the slope soil to the surrounding water. The experiment simulated the transport of Cryptosporidium surrogate, carboxylate YG polystyrene, influenced by the rainfall intensity, rainfall pattern, soil type, and land slope, from the soil to water, to understand the transport of Cryptosporidium surrogate under these different conditions. The results showed that the transport of Cryptosporidium surrogate was affected by the surface runoff factors, that is, the high rainfall intensity, high rainfall frequency, steep slope and high sand content soil that resulted in the high transport of the Cryptosporidium surrogate.
\end{abstract}

\section{INTRODUCTION}

Cryptosporidium is a pathogenic microorganism that induces diarrhoea and vomiting (Yue \& Ni 2014, Wang 1993), which has serious implications for the development of recreational water body activities and human health.

In recent years, an outbreak of Cryptosporidium caused by the medium of water has been reported in countries such as the UK and the United States. After the reported cases of human infection with Cryptosporidium in 1976 (Ryan et al. 2003), more than 90 countries appeared in succession with regard to report about Cryptosporidium infection (Wu et al. 2006). In the United States, about 2 per cent of the population was tested positive for Cryptosporidium. Each year, of every 150000 patients with diarrhoea, about 30000 are caused by Cryptosporidium infection. In 1984 and 1987, the United States experienced two outbreaks of cryptosporidia, and the total number of cases was 15,000. In 1993 at Minkwakee, Wisconsin, the largest outbreak of cryptosporidia was reported with up to 400,000 people infected and it has remained to be the highest and most serious case of Cryptosporidium outbreak up to date. In 1987, the first cases of Cryptosporidium infection in humans were diagnosed in Nanjing, China.
The main transmission channels of Cryptosporidium are direct contact of the skin such as swimming and diving, as well as through food and water. According to the survey, the infection rate of swimming in lakes and swimming pools is much higher than that of eating contaminated food (Zhang \& Jiang 2001, Wang \& Yan 2008, Wu \& Li 2005). Kong et al. (2017) reported that the risk of Cryptosporidium infection in a certain lake in China was 18 cases/10,000 person.

Therefore, adequately understanding the transport pathway of Cryptosporidium in lakes is of great significance to control surface water quality and maintenance of human health. This experiment simulated the different conditions of rainfall, rainfall patterns, soil types and soil slope study on the transport of Cryptosporidium surrogate from slope soil to the surface water, to reveal the interface between soil and water transportation law of Cryptosporidium and key function parameters.

\section{MATERIALS AND METHODS}

\section{The Carboxylate YG Polystyrene Microspheres}

Cryptosporidium is detrimental to human health, so it is diffi- 
cult to fully guarantee that its leakage would pose a threat to the operator and the environment. Therefore, the experiment will be carried out with the surrogate of Cryptosporidium oocysts, the polystyrene microspheres, which have been proved to be effective as a surrogate for Cryptosporidium oocysts (Lu et al. 2013, Lu et al. 2014a, Lu et al. 2014b, Lu et al. 2016). In this experiment, Fluoresbrite ${ }^{\mathrm{TM}}$ Carboxylate YG polystyrene microspheres (Polysciences, Inc., Warrington, PA, USA) with a diameter of $4.869 \mu \mathrm{m}$ were used as a model particle surrogate for Cryptosporidium. The microsphere has the same shape, diameter, density and surface charge as the true Cryptosporidium.

In this study, the microspheres were counted by fluorescence microscope. The collected experimental samples were put into a centrifugal machine, shocked and homogeneously mixed, then filtered by using a circular filter with an aperture of $2.5 \mu \mathrm{m}$ and a diameter of $20 \mathrm{~mm}$. The filter was placed in the slide, and after fixation of the slide, it was observed with the fluorescence microscope (Zhang et al. 2014).

\section{The Experimental Setup}

The experimental device is composed of $50 \mathrm{~cm}$ slope organic glass tank and rainfall device, as shown in Fig. 1. The organic glass tank simulates the soil slope of the surface water body. The lowest point has the sampling port, and the surface runoff is collected to analyse the contents of the surrogate for Cryptosporidium. Rainfall devices include rainfall sprinkler, circulating pump, collecting bottle and catheter, etc. Note that rainfall is adjustable when using the organic glass tank.

\section{The Experimental Method}

The effects of rainfall intensity, rainfall pattern, soil type and land slope on the transport of Cryptosporidium were simulated. According to the rainfall situation in Xuzhou, the rainfall intensity was simulated, which can be categorized into three modes: heavy rain $(21 \mathrm{~mm} / 12 \mathrm{~h})$, rainstorm $(42 \mathrm{~mm} / 12 \mathrm{~h})$ and heavy rainstorm $(63 \mathrm{~mm} / 12 \mathrm{~h})(\mathrm{Xu}$ et al. 2008). The sand slope was $30^{\circ} \mathrm{C}$. The $1 \mathrm{~mL}$ surrogate (the Cryptosporidium oocysts' content was $100 / \mathrm{mL}$ ) was mixed into the soil. Then the rainfall intensity was adjusted by rainfall device, respectively $21 \mathrm{~mm} / 12 \mathrm{~h}, 42 \mathrm{~mm} / 12 \mathrm{~h}$, and $63 \mathrm{~mm} / 12 \mathrm{~h}$, to determine the transport quantity of Cryptosporidium in 30 minutes at different rainfall intensity. The rainfall pattern was set to three modes while the total rainfall duration was 4 hours. The three modes are further elaborated below.

Rainfall was $2 \mathrm{~h}$, stopped $2 \mathrm{~h}$;

Rainfall was $1 \mathrm{~h}$, intermitted $1 \mathrm{~h}$, and repeated once;

Rainfall was $0.5 \mathrm{~h}$, intermitted $0.5 \mathrm{~h}$, and repeated 4 times.

Under the same rainfall intensity $(21 \mathrm{~mm} / 12 \mathrm{~h})$, the soil was divided into four types of sand content, which was $100 \%$, $80 \%, 60 \%$ and $40 \%$ respectively, and matched with granulated sand grains and clay. Under the same rainfall intensity $(21 \mathrm{~mm} / 12 \mathrm{~h})$, the land slope was $10^{\circ}, 20^{\circ}, 30^{\circ}$.

\section{RESULTS AND DISCUSSION}

\section{Effect of Rainfall Intensity on the Transport of Microspheres}

The effects of rainfall intensity on the transport of microspheres are shown in Fig. 2. The transport of microspheres was positively correlated with rainfall intensity. The escalation of rainfall intensity increased the runoff through the sandy soil. In the case of constant soil pore size, the increase of runoff in unit time resulted in the increased number of the microspheres that were transported.

\section{Effects of Rainfall Patterns on the Transport of Microspheres}

The effects of rainfall patterns on the transport of microspheres are shown in Fig. 3. The higher the rainfall frequency was, the greater the total transport of microspheres was. In the

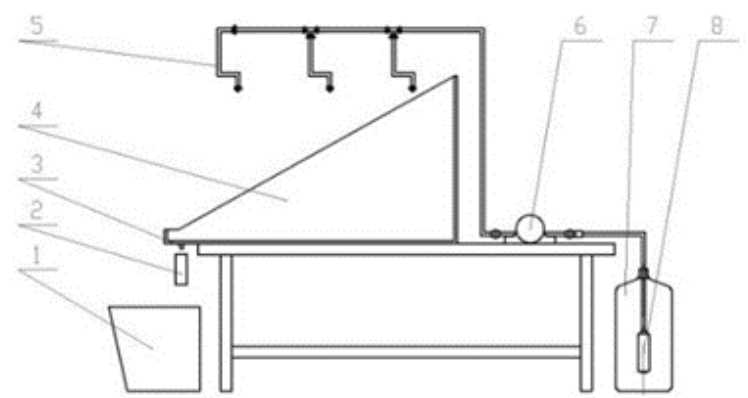

Fig. 1: Schematic diagram of the experimental model device (1. Wastewater tank, 2. Sampling pipe, 3. Sampling, 4. Soil tank, 5. Sprayer, 6. Pump, 7. Clean water tank, 8. Filter). 
case of high rainfall frequency, the rainfall was stopped, but the runoff persisted, which caused the microspheres to continue to transport with the surface runoff when the short-term rainfall was suspended.

\section{Effects of Soil Type on the Transport of Microspheres}

The effects of soil type on the transport of microspheres are shown in Fig. 4. The amount of sand contained in soil was positively proportional to the transport of microspheres. The higher the sediment concentration was, the greater the transport of the microspheres was. The underlying reason is that absorption and water-retention of sandy soil is indisputably smaller than clay.

\section{Effects of Land Slope on the Transport of Microspheres}

The effects of land slope on the transport of microspheres are shown in Fig. 5. The augmentation of land slope decreases the water storage capacity of river slope, and the amount of water in the soil can be reduced. When the rain went through flat slope (e.g., $10^{\circ}$ slope), the quantity of the trapped water was more than the steep slope, resulting from the less runoff,

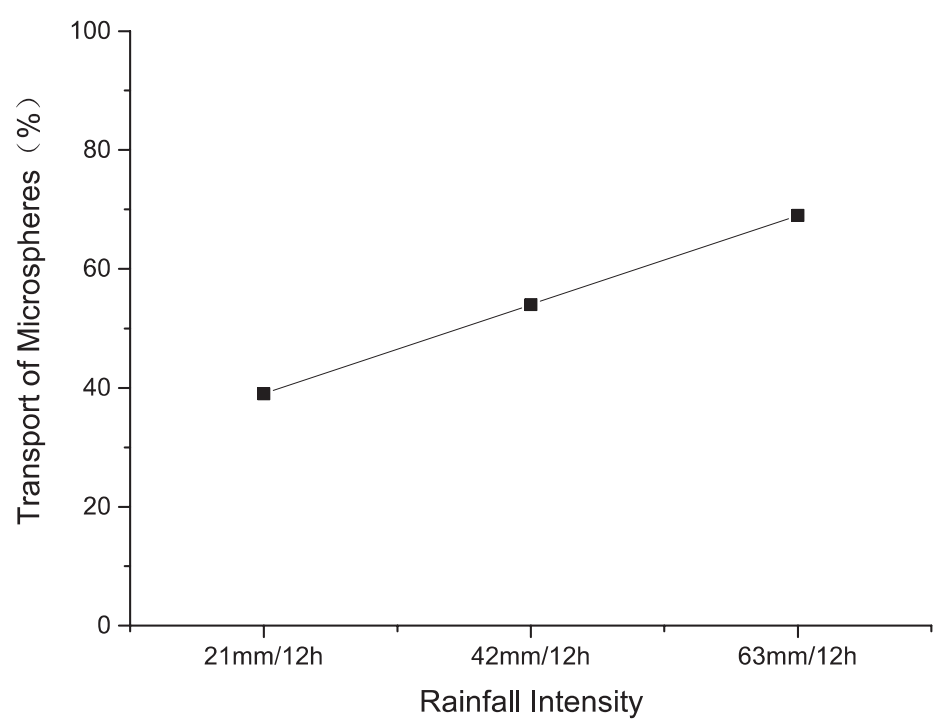

Fig. 2: Influence of rainfall intensity.

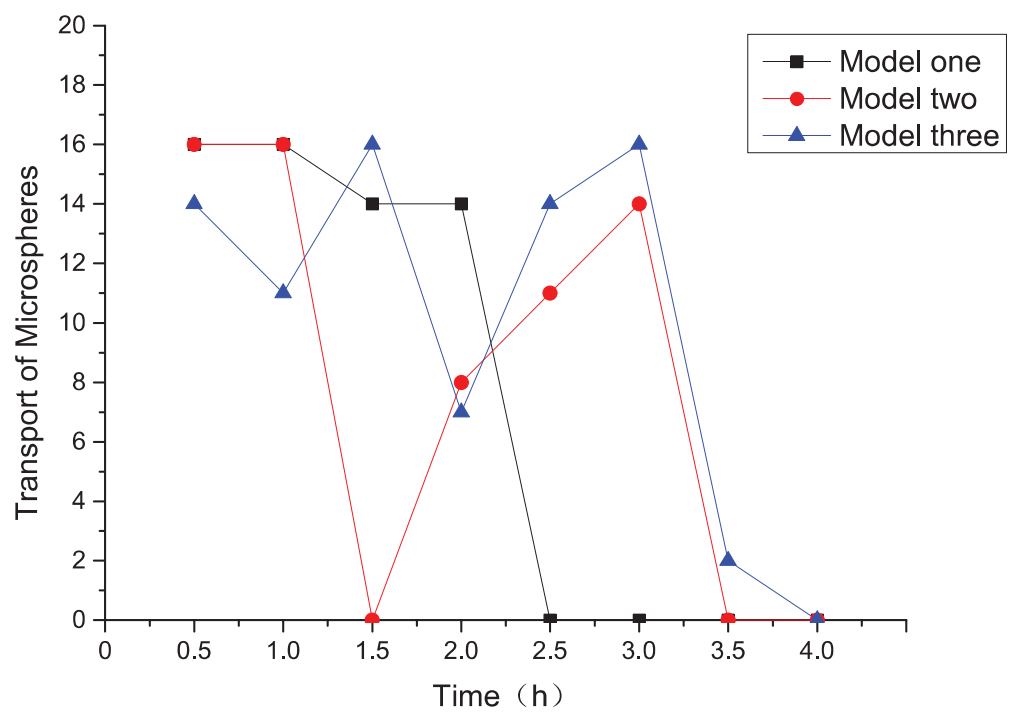

Fig. 3: The number of microspheres transported under the influence of rainfall pattern. 


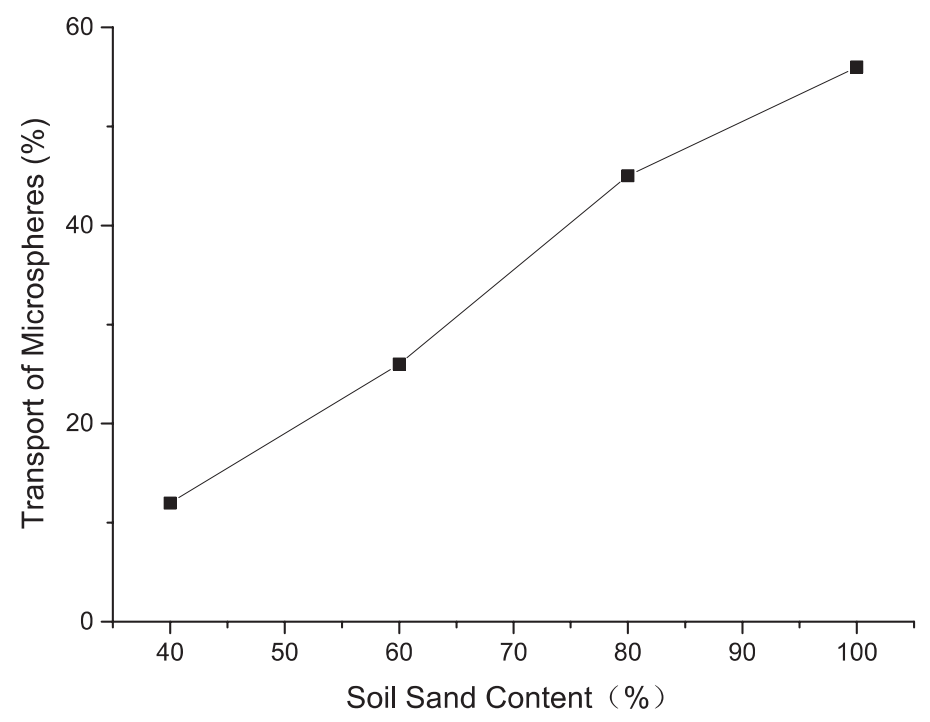

Fig. 4: Per cent of microspheres transported under the influence of soil type.

and the transport of microspheres was relatively minute. With the increase of slope, the amount of runoff increased, and the transport of microspheres was increased as well.

\section{CONCLUSIONS}

The soil is a sink of faecal pollutants such as Cryptosporidium oocyst. The transport of Cryptosporidium surrogate, which is caused by rainfall, to the overlying water of the surrounding surface rivers or lakes, is the main passway of the soil-water transport of Cryptosporidium surrogate. The transport of Cryptosporidium surrogate was more influenced by the surface runoff factors, the high rainfall intensity, frequent rainfall frequency, steep slope and high sand content of the soil. The results could serve as a significant guide to control the transport of Cryptosporidium from the slope to the overlying water.

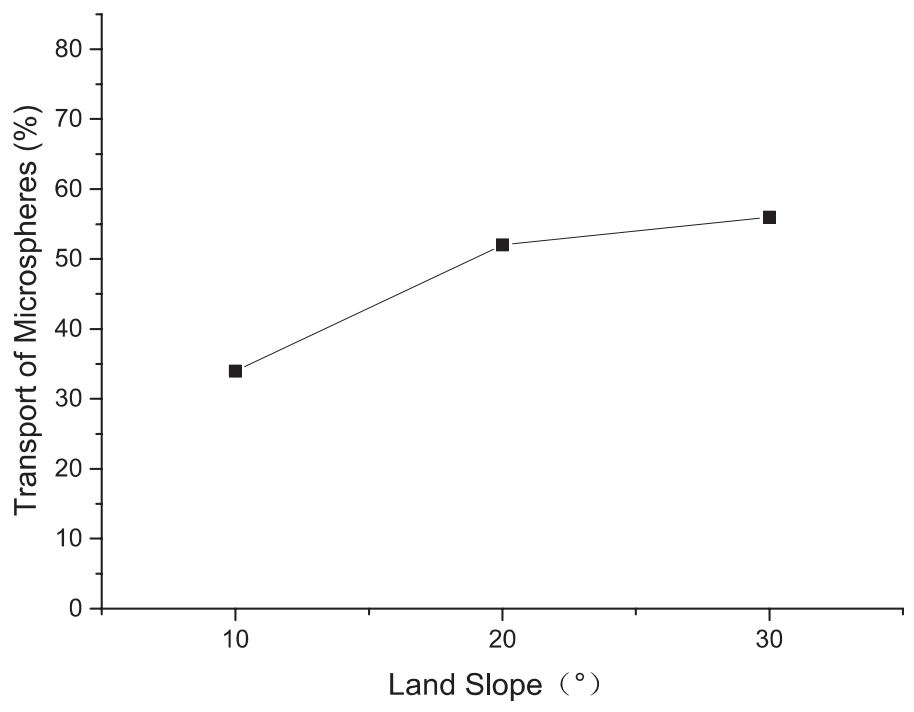

Fig. 5: Per cent of microspheres transported under the influence of land slope. 


\section{ACKNOWLEDGEMENT}

This project was supported by the Foundation of Ministry of Housing and Urban-Rural Development of China (2016K4-076) the Foundation of Jiangsu Provincial Department of Housing and Urban-Rural Development(2017ZD013) and Provincial University Natural Science Fund of Jiangsu (16KJB610016).

\section{REFERENCES}

Kong, Y., Lu, P., Tao Yuan., Niu, J., Li, Z. and Yang, B. 2017. Cryptosporidium contamination and attributed risks in Yunlong Lake in Xuzhou, China. Canadian Journal of Infectious of Infectious Diseases and Medical Microbiology, 1-6.

Lu, P., Yuan, T., Feng, Q., Xu, A. and Li J. 2013. Review of swimming-associated Cryptosporidiosis and Cryptosporidium oocysts removals from swimming pools. Water Quality Research Journal of Canada, 48(1): 30-39.

Lu, P., Yuan, T., Feng, Q., Li, J., Sun, Y. and Li, T. 2014. Pilot-scale studies on Cryptosporidium oocysts surrogate removal from swimming pool. Chinese Journal of Environmental Engineering, 8(7): 2822-2826.

Lu, P., Yuan, T. and Feng, Q. 2014. Using of poly diallyl dimethylammonium chloride for removal Cryptosporidium from the public recreational water venue. Journal of Chemical and Pharmaceutical Research, 6(6): 39-43. Lu, P., Yuan, T. and Zhang, B. 2016. Cryptosporidium removal from runoff by active carbon filter with goal gangue. Journal of Chemistry, 1-3.

Ryan, U.M., Samarasinghe, B., Read, C., Buddle, J.R., Robertson, I.D. and Thompson, R.C.A. 2003. Identification of a novel Cryptosporidium genotype in pigs. App. Enviro. Microbiol., 69(7): 3970-3974.

Wang, G. 1993. Human Cryptosporidiosis research progress in our country. The Journal of Epidemiology, 14(1): 52.

Wang, L. and Yan, G. 2008. Human Cryptosporidiosis epidemiological and clinical studies. Chinese Journal of Pathogen Biology, 12(3): 953-956.

Wu, L., Chen, S. and Cao J. 2006. The popularity of Cryptosporidiosis and diagnosis research progress. Parasitic Disease International Medical Journal, 30(5): 277-281.

Wu, L. and Li, P. 2005. The research progress of Cryptosporidium pathogenesis. The Chinese Veterinary Parasitic Diseases, 13(3): 28-33.

Xu,L., Zhang, Q. Xu, J., Jiang, H. and Huang, L. 2008. Different rainfall intensity on nutrient vertical transport and leaching rate. Journal of Soil, 45(3): 437-444

Yue, H. and Ni, C. 2014. Cryptosporidium and its research progress of the disease. Jiangsu Preventive Medicine, 25(5): 44-46.

Zhang, L. and Jiang, J. 2001. Research progress of Cryptosporidium and Cryptosporidiosis. Journal of Insect Parasites and Medicine, 8(3): 184-192.

Zhang, W., Xu, A. and Zhang, R. 2014. Soil classification research review and revision of the Chinese soil classification systems. China's Agricultural Science, 47(16): 3214-3230. 